\title{
Corrigendum: Lactate-mediated glia-neuronal signalling in the mammalian brain
}

\author{
F. Tang, S. Lane, A. Korsak, J.F.R. Paton, A.V. Gourine, S. Kasparov \& A.G. Teschemacher
}

Nature Communications 5:3284 doi: 10.1038/ncomms4284 (2014); Published 11 Feb 2014; Updated 28 May 2014

While this Article was under consideration, Bozzo et al. published their findings on the inhibitory effects of L-lactate on cortical neuron activity. This paper should have been cited in the Discussion as follows:

Recently L-lactate has been reported to inhibit activity of cultured cortical neurons (Bozzo et al. 2013). This effect was consistent with previously reported properties of the GPR81 (HCA1) receptor ${ }^{44-47}$. Specifically, the IC50 for L-lactate was estimated to be $\sim 4.2$ mM, and the effect was mediated by $\mathrm{G}_{\mathrm{i}}$-proteins based on the sensitivity to pertussis toxin.

Bozzo, L., Puyal, J., \& Chatton, J. Y. Lactate modulates the activity of primary cortical neurons through a receptor-mediated pathway. PLoS ONE 8, e71721 (2013). 